\title{
Attitude towards psychiatric treatment and referral pattern in the University of Maiduguri Teaching Hospital- A preliminary report
}

\author{
MA Wakil', IW Abdul', MS Jidda', OBeida' ${ }^{2}$, A Ahidjo³, FK Salawu4, JO Abdulmalik ${ }^{5}$ \\ 1Department of Mental Health, College of Medical Sciences, University of Maiduguri, Maiduguri, Borno State, Nigeria \\ 2Department of Mental Health, Federal Neuro-psychiatric Hospital, Maiduguri, Borno State, Nigeria \\ ${ }^{3}$ Department of Radiology, College of Medical Sciences, University of Maiduguri, Maiduguri, Borno State, Nigeria \\ ${ }^{4}$ Department of Medicine, Federal Medical Centre, Yola, Adamawa State, Nigeria \\ ${ }^{5}$ Department of Psychiatry, University College Hospital, Ibadan, Oyo State, Nigeria
}

\begin{abstract}
Objective: There is a paucity of literature on consultation-liaison psychiatry, in northern Nigeria. This study aimed to determine both the pattern of psychiatric referrals, and the attitudes of doctors toward the treatment of mental disorders in a teaching hospital, in northeast Nigeria. Method: In this cross-sectional survey, we used a modified version of the self-rated Kumar 12-item questionnaire and a basic socio-demographic questionnaire to assess a non-random convenient sample of 100 postgraduate resident doctors (with a response rate of 70\%) from the University of Maiduguri Teaching Hospital (UMTH). We subjected the data obtained to descriptive statistical analysis, using EPI info (2003), to report averages. Results: A relatively low percentage (57.1\%) of doctors acknowledged treating patients with mental disorders in their practice, with a higher proportion acknowledging referral (75\%). Nearly one in five (17.6\%) of the respondents were unaware that patients with functional illness could have psychological disorders. We found more awareness for psychotherapy (44.1\%) than other non-pharmacological treatment interventions, while 10.3\% were ignorant of non-pharmacological forms of treatment for psychological problems. Conclusion: Although this is a preliminary report, the research reported here demonstrated that doctors in the teaching hospital concerned recognized the need for psychiatric consultation and referral. It is difficult to draw further conclusions because of the limitations of this study.
\end{abstract}

Keywords: Consultation; Liaison; Psychiatry; northern Nigeria

Received: 16-01-2012

Accepted: 14-05-2012

doi: http://dx.doi.org/10.4314/ajpsy.v16i3.24

\section{Introduction}

Studies on teaching hospital inpatients have reported a low rate of psychiatric referral and various barriers to treatment. ${ }^{1,2}$ Stigma and negative attitudes among other doctors significantly affects the practice of consultation-liaison - an area of psychiatric practice which involves consultation and collaboration with non-psychiatric physicians and other health workers in all types of medical care settings, including teaching hospitals. ${ }^{3}$

\section{Correspondence}

Dr. A Wakil

Department of Mental Health, College of Medical Sciences,

University of Maiduguri, Maiduguri, Borno State, Nigeria

email: bawaki@yahoo.com
Psychiatric and emotional problems are often under recognized and therefore, poorly treated. Psychological factors may be major determinants of the presentation as well as consequences of physical illness and medically unexplained symptoms. They make up a large proportion of clinical practice in both primary and secondary care. ${ }^{4}$ It is therefore, essential for medical professionals to understand and work closely with the psychiatrist to identify and resolve these problems in their patients. Furthermore,

psychopathology that may be associated with physical illness often presents as an emergency to these doctors. ${ }^{1,2}$

Some evidence from studies of attitudes of physicians and surgeons in a teaching hospital indicates low referral rates ${ }^{2-5}$ specifically related to stigma ${ }^{4}$, severity of illness and past psychiatric history. ${ }^{3}$ Both the patient and the physician play a 
role in referrals, and one study found patients admitted to a general hospital with emotional disorders accounted for $25 \%$ of all admissions but referrals to consultation-liaison services rarely exceed $10 \%{ }^{2}$ Similar studies in general practice found non-recognition of the extent of psychiatric morbidity among patients, stigma, concerns about the effect of psychiatric referral on self-esteem of patients, not being sure of how beneficial such referral could be, and difficulty in securing psychiatric services as all affecting the referral decisions of most physicians. ${ }^{5}$ Some studies explored family physicians' attitudes toward psychiatry and identified two reasons for non-referral: firstly concerns about the effectiveness of psychiatric treatment and secondly stigma for the patient. They found the use of psychotropic drugs often necessary, but also recommended psychotherapy and combined approaches. ${ }^{6}$

However, the commonly held belief that failure to recognize psychiatric morbidity accounts for the low rate of referrals to various psychiatric liaison services may not be true. A study of primary care provider referral patterns for patients with psychosocial problems looked at factors that influence mental health referral. ${ }^{5}$ Six hundred fifty (16\%) of 4012 patients with psychosocial problems were referred at the initial visit. Factors associated with the likelihood of referral included patient factors (severity, type of problem, academic difficulties, and prior mental health service use) and family factors (mental health referral of parent); however, none of the provider factors was significant. While clinicians reported frequent barriers to referral and mental health services in the general background survey, these factors rarely influenced individual management decisions. ${ }^{5}$

A more recent study of treatment options in psychiatry suggested the widespread use of listening, reassuring and discussing anxiety by hospital doctors was heartening. The study did not address the detail of these interventions, but at the very least, this seemed to indicate awareness of psychotherapeutic techniques and their efficacy. ${ }^{9}$ Earlier studies found hospital doctors rely on pharmacological rather than the psychological treatments. ${ }^{10-12}$

Data on consultation-liaison psychiatry remains limited in northern Nigeria. A previous study in the region found the highest rate of referral came from the internal medicine department. The most common psychiatric disorders referred were acute organic brain syndrome (32\%) and neurologic disorders (15\%). Two-thirds of the patients received medication and the remaining one-third had counseling. Half of the discharged outpatients defaulted on their clinic appointments. ${ }^{3}$

Information from other geopolitical regions of the country could also contribute to the development of policy on consultation-liaison psychiatry in Nigeria. This has prompted the present study, to assess the attitudes toward treatment and referral in a teaching hospital, in northeast Nigeria.

\section{Method}

\section{Site/Sample}

The cross-sectional study was conducted at the University of Maiduguri Teaching Hospital (UMTH), Maiduguri, Borno state, Nigeria. It is the first teaching hospital in the northeast geopolitical zone of the country, and it is designated the "Center of Excellence" in Infectious Diseases and Immunology by the
Federal Government. It serves as a referral centre for the Northeast (comprising of present day Borno, Yobe, Adamawa, Gombe, Bauchi and Taraba states) political zone of Nigeria, the republic of Chad, eastern portion of Niger Republic and northern Cameroons. Furthermore, the only well organized psychiatric services are rendered by the Federal Neuropsychiatric Hospital, Maiduguri. A sample of 100 out of 123 (81.3\%) postgraduate resident doctors from the hospital was eligible for inclusion in the study.

\section{Instruments}

They were assessed using an existing self-rated Kumar 12item questionnaire ${ }^{10}$ (See Appendix 1), and basic socio-demographic data (age, sex, etc.) were obtained for each doctor. We modified the questionnaire (comprised of questions covering attitude to psychiatry, assessment of psychological problems, referral to psychiatrists and the treatment of psychological disorders) to correct for vague or ambiguous questions such as the wording of questionnaire items $1,2,3,4,6$, and 10 , since the framing of the questions may affect the results.

\section{Ethics}

The Ethics and Research Board of the UMTH provided ethical approval and informed consent obtained from the individual doctors. Respondents were aware that the interview and the study were unconnected with the institution but also that participation in this study was voluntary. Confidentiality was ensured by not recording the name of the participant anywhere on the survey.

\section{Analysis}

We subjected the data obtained to descriptive statistical analysis, using EPI info (2003), to report averages.

\section{Results}

Gender, age, specialty of the respondents, knowledge about treatment and referral of psychological problems

Seventy of the 100 post-graduate doctors non randomly sampled (out of 123 who could have been sampled) participated in the study, giving a 70.0\% response rate. Therefore out of 123, a sample of 70 (56,9\%) was included in the study. Two (2.0\%) doctors refused to participate in the study; 12 (12.0\%) refused to answer the questions; 3 (3.0\%) lost the questionnaires and 13 (13.0\%) did not return the questionnaires.

Males constituted about two thirds of the participants (74.3\%), aged between 25 and 45 years. Respondents had a mean age of 32.6 ( \pm 4.1 years); with the majority (95.7\%), aged less than 40 years. Twenty (32.9\%) doctors were specializing in internal medicine; 15 (21.4\%) surgery, and 12 (17.1\%) Obstetrics \& Gynaecology (O\&G), (Table I).

Fourteen (20\%) of the respondents did not see psychiatric cases. Of the 56 who did see psychiatric cases more than half (57.1\%) of these respondents treated such patients. Whilst a figure of $75 \%$ was obtained for those who referred cases it should be noted that this finding was based on response from only 32 of the 70 respondents.. Within the group acknowledging that patients with medical illness could have psychological conditions (84\%), 10.3\% did not know of other treatment options beyond pharmacological. Whilst 
Table I. Gender, age and specialty of the respondents, knowledge about treatments and referral of psychological problems

\begin{tabular}{|lcc|}
\hline Variable & Frequency & (\%) \\
\hline Gender & & \\
Male & 52 & 74.3 \\
Female & 18 & 25.7 \\
Total & 70 & 100.0 \\
\hline Age & & \\
$20-29$ & 17 & 24.3 \\
$30-39$ & 50 & 71.4 \\
$40+$ & 3 & 4.3 \\
Total & 70 & 100.0
\end{tabular}

\section{Speciality}

Intemal Medicine $\quad 23 \quad 32.9$

$\begin{array}{lll}\text { Ophthalmology } & 2 & 2.9\end{array}$

$\begin{array}{lll}\text { Pediatrics } & 6 & 8.6\end{array}$

Radiology

Surgery

General Practitioner (Family Medicine)

7

15

5

Total

70

10.0

21.4

7.1

100.0

Did you see psychiatric cases?

$\begin{array}{lcc}\text { Yes } & 56 & 80.0 \\ \text { No } & 14 & 20.0 \\ \text { Total } & 70 & 100.0\end{array}$

If 'Yes' what \%?

Less than 5

$5-10$

10-20

More than 20

Total

37

66.1

26.8

5.3

1.8

100.0

Do you treat some psychiatric cases?

$\begin{array}{lcc}\text { Yes } & 32 & 57.1 \\ \text { No } & 24 & 42.9 \\ \text { Total } & 56^{\mathrm{a}} & 100.0\end{array}$

Do you refer some psychiatric cases?

$\begin{array}{lcc}\text { Yes } & 24 & 75.0 \\ \text { No } & 8 & 25.0 \\ \text { Total } & 32^{\mathrm{a}} & 100.0\end{array}$

Are you aware the patients with medical illnesses could have associated psychological conditions?

$\begin{array}{lcc}\text { Yes } & 56 & 82.4 \\ \text { No } & 12 & 17.6 \\ \text { Total } & 68^{\mathrm{a}} & 100.0\end{array}$

Apart from drugs, do you know other treatments for psychiatric patients?

$\begin{array}{lcc}\text { Yes } & 61 & 89.7 \\ \text { No } & 7 & 10.3 \\ \text { Total } & 68^{\mathrm{a}} & 100.0\end{array}$

Treatments that exist apart from drugs for psychiatric patients include:

Psychotherapy

$\begin{array}{lll}\text { Counselling } & 26 & 27.1\end{array}$

Behaviour therapy

Electro-convulsive therapy

Occupational therapy

Social therapy

Religious therapy

Total

100.0 respondents had greater awareness for psychotherapy (44.1\%), as a non-pharmacological treatment modality, they had less awareness for other forms of treatment such as electroconvulsive therapy (10.2\%) and social therapy (6.8\%), specifically associated with psychiatry (Table I).

\section{Knowledge about, attitude towards psychosocial care and} treatment of psychological problems

$91.8 \%$ of the respondents agreed with the opinion that psychological factors influence the presentation, course and outcome of physical illness; $45.7 \%$ prescribed psychotropic medication and $41.4 \%$ disagreed with the opinion of posting a psychiatrist to a primary health centre.

95.7\% respondents saw psychiatry as a valid medical discipline, but only $43.7 \%$ felt it should have a separate department, and $51.6 \%$ felt it should be practiced in a general medical set-up. Furthermore, 95.7\% of the respondents agreed psychiatry should be taught in undergraduate medical courses, but only 41.1\% wanted a separate paper (theory/clinical) of psychiatry during the undergraduate examinations, (Table II).

\section{Discussion}

The current study comprised a small non-random sample size of 100 (out of 123) with a response rate of 70\%. Doctors in this teaching hospital recognized the need for psychiatric help, with 75\% reporting referral to Psychiatry, but also demonstrated a capacity to treat with $57.1 \%$ who acknowledged treating patients with mental disorders in their practice.

Awareness of the relevance of psychological factors associated with physical illness and a high referral rate in this sample is comparable to results obtained in the developed countries. ${ }^{9}$ It contrasted with the low rates of referral found in a prospective study of psychotropic drug use in a similar setting using standardized research instruments, in northern Nigeria $^{13}$ and a study of attitudes of primary care physicians to psychiatric referral using a structured questionnaire, in southern Nigeria. ${ }^{14}$ The present study did not assess reasons for non-referral but pejorative attitudes to psychiatric referrals have previously been the reported. ${ }^{10}$

The present study did not address the details of treatment options in psychiatry, but found a relatively high psychotropic prescription rate (45.7\%) and at the very least findings indicated an awareness of psychotherapy (44.1\%). It also contrasted with the results of a previous survey, in a similar setting which found a lower psychotropic prescription rate (33.1\%). and another study in which hospital doctors appeared to rely on pharmacological rather than psychological treatments. ${ }^{13}$

There are some caveats to the findings. Firstly, selfreporting instead of actually counting referrals makes it difficult to confirm the results and thus extrapolate to clinical practice in other settings. Further, a mixed method approach that included key informant interviews would have allowed a more nuanced qualitative exploration of attitudes towards psychiatry. Future studies using standardized research instruments might address the response bias associated with results of a questionnaire. Secondly, the findings of the study conducted at a single centre may not apply to other hospital settings. 
Table II. Shows knowledge about, attitude towards psychosocial care and treatment of psychological problems

\begin{tabular}{|lcc|}
\hline Statement & Frequency & (\%) \\
\hline Do you prescribe psychotropic medications? & & \\
Yes & 32 & 45.7 \\
No & 38 & 54.3 \\
Total & 70 & 100.0
\end{tabular}

Psychological factors can influence the presentation, course and outcome of physical illness

$\begin{array}{lcc}\text { Yes } & 56 & 91.8 \\ \text { No } & 5 & 8.2 \\ \text { Total } & 61^{\mathrm{a}} & 100.0 \\ & & \\ \text { Psychiatry is a valid medical discipline } & & \\ \text { Yes } & 67 & 95.7 \\ \text { No } & 3 & 4.3 \\ \text { Total } & 70 & 100.0\end{array}$

If 'No' chose the specialty?

$\begin{array}{lcc}\text { Intemal Medicine } & 4 & 80 \\ \text { Neurology } & 1 & 20 \\ \text { Total } & 5^{\mathrm{a}} & 100.0\end{array}$

Psychiatry should be taught in undergraduate MBBS course

$\begin{array}{lcc}\text { Yes } & 67 & 95.7 \\ \text { No } & 3 & 4.3 \\ \text { Total } & 70 & 100.0\end{array}$

In your view should there be a separate paper (theory/clinical) of psychiatry during the undergraduate examination?

$\begin{array}{lcc}\text { Yes } & 29 & 41.4 \\ \text { No } & 41 & 58.6 \\ \text { Total } & 70 & 100.0\end{array}$

Psychiatry should be practiced in a separate psychiatric set-up

$\begin{array}{llc}\text { Yes } & 40 & 58.8 \\ \text { No } & 28 & 41.2 \\ \text { Total } & 68^{\mathrm{a}} & 100.0\end{array}$

Psychiatry should be practiced in a General Medical set-up

$\begin{array}{lcc}\text { Yes } & 33 & 51.6 \\ \text { No } & 31 & 48.4 \\ \text { Total } & 64^{\mathrm{a}} & 100.0\end{array}$

Yes, under a separate department (specify)

$\begin{array}{lll}\text { Integrated with Internal Medicine } & 8 & 50.0\end{array}$

Neurology

Psychiatry

Total

7

$16^{a}$

6.3

43.7

100.0

The posting of a psychiatrist, to a Primary Health Care Center is all right

$\begin{array}{llc}\text { Yes } & 41 & 58.6 \\ \text { No } & 29 & 41.4 \\ \text { Total } & 70 & 100.0\end{array}$

Total

70

100.0

aSome respondents did not answer some questions, thus accounting for less than 70

\section{Conclusion}

Psychiatric referral and psychotropic prescription rates in this study suggest levels of awareness of psychiatric disorders as well as confidence to initiate treatment in such instances. This is an interesting, and potentially positive, finding in a setting of scarce psychiatric resources. The findings suggest that when doctors recognize psychiatric symptoms, they may initiate treatment but also request for consultation to the psychiatrist via the referral system. The current study used a questionnaire instead of actual hospital records. It may be interesting to compare subsequent findings to the current findings, using standardized instruments and patients' records of documented referral patterns over time. Although this is a preliminary study, the findings clearly indicate that doctors in the teaching hospital studied recognize the need for psychiatric consultation, have a level of confidence in prescribing psychotropic medication, are aware of psychotherapy as an intervention and do consider psychological factors relevant. It is difficult to draw further conclusions because of the limitations of this study.

\section{Acknowledgement}

We gratefully acknowledge the postgraduate resident doctors at the University of Maiduguri, Teaching Hospital for completing the questionnaire. Similarly, we would like to express our profound gratitude to Prof. Jennifer Swann, Department of Biological Sciences, 111 Research Drive, Lehigh University Bethlehem, PA 18104, 610- 758-5484, for her mentorship and encouragement in the preparation of this document.

\section{References}

1. Ruddy R, House A. A standard liaison psychiatry service structureA study of the liaison psychiatric services within six strategic health authorities. Psychiatric Bulletin 2003; 27:457-460.

2. Lipsitt DR. Helping Primary Care Physicians Make Psychiatric Referrals. Some Practical Considerations. Psychiatric Times 2010; Vol. 27 No. 12.

3. Ajiboye PO, Adelekan ML. A prospective analysis of inpatient consultation-liaison psychiatry in a Nigerian teaching Hospital. East African Medical Journal 2004; Vol. 81 (12):620-625.

4. Geoffrey G, Mayou RA. Liaison psychiatry or psychological medicine? British Journal of Psychiatry 2003; 183:5-7.

5. Rushton J, Bruckman D, Kelleher K. Primary Care Referral of Children with Psychosocial Problems. Arch Pediatr Adolesc Med 2002; 156:592-598.

6. http://www.wpanet.org/detail.php?section_id=7\&content_id=922

7. Leighton AH, Lambo TA, Hughes CC, Leighton DC, Murphy JM, Macklin DB. Psychiatric disorders in West Africa. Am J Psychiatry 1963; 120:521-7.

8. Hartley D, Korsea N, Bird D, Agger M. Management of patients with depression by rural primary care practitioners. Arch Fam Med 1998; 7:139-145.

9. Morgan JF, Killoughery M. Hospital doctors' management of psychological problems- Mayou \& Smith revisited. British Journal of Psychiatry 2003; 182:153-157.

10. Kumar A, Goyal U, Ganesh KS. Attitudes of postgraduate resident doctors toward psychiatry. Indian Medical Journal 2001; 43 (2):1-5.

11. Raynes NV. Factors affecting the prescribing of psychotropic drugs in general practice consultations, Psychological Medicine 1979; 9:671-679

12. Tyrer P, Murphy S. The place of benzodiazepines in psychiatric practice. British Journal of Psychiatry 1987; 151:71 9-723.

13. Abiodun OA, Ogunremi OO. Psychotropic drug use in medical and surgical wards of a teaching hospital in northern Nigeria. British Journal of Psychiatry 1991; 159:570-578.

14. Aghanwa HS, Morakinyo O, Aina O F. Consultation-liaison psychiatry in a general hospital setting, in West Africa. East Afr Med J 1996; 73(2):133-6. 


\section{Appendix I: Questionnaire for assessing attitude towards psychiatry}

I know that by returning the/this questionnaire, I am giving my informed consent as a participating volunteer in this study. I also appreciate the benefits that might be realized from the successful completion of this study. I realize that I have the right to refuse to participate, and I have the right to withdraw from participation in this study without coercion or prejudice.

Age,

Sex,

Specialty (Department)

Wherever applicable, please tick or give your opinion in a few words:

1. Do you see some psychiatric cases?

If 'Yes', show percentage,

a. Less than 5

b. $5-10$

c. $10-20$

d. More than 20

2. Do you treat some psychiatry cases?

3. Do you refer some psychiatry cases?

4. Patients with medical illnesses could have associated psychological conditions,

5. Apart from drugs, there are other treatment options for psychiatric patients,

If 'Yes', recommend the treatment options,

6. Do you prescribe psychotropic medications?

If 'Yes' specify,

a. Recommend the class of psychotropic medication

b. Reason(s) for prescribing psychotropic medication

c. Duration of treatment with psychotropic medication

7. Psychological factors affect the presentation, course and outcome of diseases,

8. Psychiatry is a valid medical specialty, is it?

9. Psychiatry should be an independent medical specialty, should it?

If 'No' under which specialty, Specify,

10. Psychiatry should be taught in undergraduate MBBS course,

If 'Yes' mention duration,

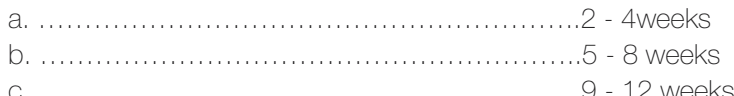

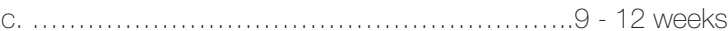

11. Psychiatry should be a separate paper (theory/clinical) during undergraduate examination,

12. Psychiatry as specialty should be practiced in a separate psychiatry set-up,

13. Psychiatry as specialty should be practiced in a general medical set-up, 